La revue La revue pour l'histoire du CNRS

12 | 2005

Les grands équipements scientifiques du CNRS

\title{
Les grands instruments de la biologie moléculaire, prémices de la médecine de demain
}

Pierre Tambourin

\section{OpenEdition}

Journals

Édition électronique

URL : https://journals.openedition.org/histoire-cnrs/1308

DOI : 10.4000/histoire-cnrs. 1308

ISSN : 1955-2408

Éditeur

CNRS Éditions

Édition imprimée

Date de publication : 3 mai 2005

ISBN : 978-2-271-06250-5

ISSN : 1298-9800

\section{Référence électronique}

Pierre Tambourin, «Les grands instruments de la biologie moléculaire, prémices de la médecine de demain », La revue pour l'histoire du CNRS [En ligne], 12 | 2005, mis en ligne le 03 mai 2007, consulté le 20 mai 2021. URL : http://journals.openedition.org/histoire-cnrs/1308 ; DOI : https://doi.org/10.4000/ histoire-cnrs.1308

Ce document a été généré automatiquement le 20 mai 2021.

Comité pour l'histoire du CNRS 


\title{
Les grands instruments de la biologie moléculaire, prémices de la médecine de demain
}

\author{
Pierre Tambourin
}

1 Longtemps réservés à la physique des particules, les besoins en très grands équipements (TGE) de la biologie deviennent urgents avec l'émergence de la génomique, science qui étudie l'ensemble des informations génétiques d'un être vivant. Ces laboratoires, inédits tant dans leur conception que dans leur taille, offrent une nouvelle vision du vivant dans ses aspects les plus intimes et promettent des révolutions conceptuelles et médicales grâce aux techniques moléculaires et informatiques qui les construisent. Ils offrent, en somme, une nouvelle dimension de l'homme qui substitue à la médecine symptomatique une médecine individualisée où l'étude du génome de l'individu permettra à la fois une prise en charge beaucoup plus spécifique du malade et un accroissement de l'efficacité du traitement chez des patients atteints du même type de lésions.

2 Deux réalisations exceptionnelles marquent, en France, le passage du $\mathrm{XIX}^{\mathrm{e}}$ au $\mathrm{XX}^{\mathrm{e}}$ siècle : la construction de l'Observatoire du Pic du Midi à partir de 1878 et celle du grand électro-aimant de Bellevue en 1920. Rien d'étonnant, en somme, que la patrie de Descartes et du pendule de Foucault soit aussi celle où l'histoire des grands instruments commence et se pérennise. Elle prendra ses habits de modernité en 1949, lorsque le physicien français Louis de Broglie propose la création d'un laboratoire scientifique d'envergure européenne, peut-être pour favoriser la reconstruction d'un continent meurtri par la guerre.

3 La mise en service en 1957 du premier accélérateur de particules du Cern ${ }^{1}$, à Genève, inaugure l'ère de la coopération scientifique internationale qui s'accomplit dans la construction de très grands équipements (TGE) et dont le rythme de création s'accélèrera au début des années 1970. La notion de TGE est donc essentiellement historique et plonge ses racines dans les mystères de l'univers et ceux de la matière, en 
particulier dans la physique des particules. L'un de ses principaux enjeux n'était-il pas, en effet, l'identification des constituants les plus élémentaires de la matière?

4 L'échelle de financement et de fonctionnement de ces grands instruments ainsi que leur degré de sensibilité et leurs exigences techniques les placent au-delà des moyens des laboratoires et, parfois, des organismes nationaux de recherche eux-mêmes. Ainsi, les laboratoires du CNRS, organisme de gestion de ces TGE de la physique, sont incapables d'en supporter seuls les coûts. La technique utilisée a donc été celle des budgets protégés qui, isolés des enveloppes classiques, sont affectés à leur construction et leurs frais de fonctionnement.

5 Ces réalisations favorisent une recherche coopérative pluri-organismes dans un contexte national, européen et international. Leur principe de fonctionnement est d'être mis gratuitement au service de la communauté scientifique pour des expériences évaluées par un comité ad hoc. Les synchrotrons, dont ceux de troisième génération comme Soleil ${ }^{2}$, sont utilisés par plusieurs milliers de chercheurs de diverses disciplines. De même, en physique gravitationnelle, le projet franco-italien Virgo $^{3}$, s'attaque à la démonstration de l'une des constructions intellectuelles les plus élaborées de la physique: l'existence, selon la théorie de la relativité générale, d'ondes gravitationnelles qui se propagent à travers l'espace, à la vitesse de la lumière.

Les nouveaux TGE

6 Jusqu'en 1995, cette notion de TGE ne concernait, de fait, que les disciplines qui s'articulaient autour de la physique ou de l'astrophysique. Or, des changements considérables sont intervenus dans le travail d'autres secteurs de la recherche, de sorte que l'office parlementaire d'évaluation des choix scientifiques et technologiques ${ }^{4}$ (OPECST) estime qu'une simple définition de ces TGE par leur coût et leur financement ou par la désignation d'un instrument unique sur un lieu unique est désormais obsolète.

7 L'exemple de la recherche en sciences de l'homme et de la société est symptomatique de cette évolution car elle a créé des besoins importants comme les bibliothèques numérisées ou les bases de données gigantesques. Même constat pour la recherche génomique et post-génomique où de grands instruments comme les centres de séquençage et les cliniques de la souris deviennent des outils " indispensables ». Or, aujourd'hui, la seule animalerie en France qui possède les caractéristiques d'un TGE est la clinique de la souris fondée à Strasbourg par le professeur Pierre Chambon.

8 L'apparition de ces nouveaux instruments très sophistiqués dans d'autres disciplines que la physique s'est accompagnée d'un changement dans les modalités de leur mise en place : désormais, ils échappent entièrement ou partiellement aux grands organismes de la recherche publique. L'OPECST souligne d'ailleurs qu'une autonomie de gestion est nécessaire aux TGE pour leur construction et leur exploitation et qu'il « serait vain et contre-productif que les plus hautes autorités de la recherche assurent le pilotage direct du lancement des TGE car la direction d'un tel projet exige une expérience de scientifique et de management et au surplus une liberté de manœuvre sinon d'action ${ }^{5}$ ». Les caractéristiques des TGE imposent donc la création de structures dotées d'une autonomie réelle, concrétisée d'ailleurs par le fait que la plupart des grands équipements sont créés ou gérés par une équipe ayant à sa tête un scientifique reconnu par ses pairs. Ainsi, lors de la création du Cern, Pierre Auger, directeur scientifique de l'Unesco, a joué un rôle essentiel. Enfin, une autre spécificité importante tient au rythme d'utilisation de ces TGE caractérisé par un service continu, 24 heures sur 24 et 7 
jours sur 7. Dans ces conditions, les mises à disposition par les organismes de recherche, de personnels soumis aux règles de la fonction publique sont parfois difficiles sinon ingérables. En conséquence, les statuts de droit privé sont mis en avant pour leur plus grande souplesse et les TGE prennent souvent les statuts juridiques de Gip (Groupement d'intérêt public) dérogatoires ou de sociétés civiles qui ont la capacité de recruter leurs personnels.

9 C'est dans ce contexte qu'au milieu des années 1990, les prémices des deux premiers grands instruments de la biologie ont vu le jour dans l'hexagone, à l'intérieur d'une structure de recherche associative, donc privée. Il s'agit du laboratoire Généthon créé par l'Association française contre les myopathies (AFM). En parallèle, un programme de recherche international démarre aux États-Unis sous le nom de Human genome project. Dès lors, l'arrivée de la génomique ${ }^{6}$ impose la mise en place de nouveaux types de laboratoires faisant évoluer la biologie d'un stade artisanal à un niveau beaucoup plus automatisé, quasi industriel. Il était donc nécessaire de trouver des solutions pour mobiliser des moyens propres à créer les structures ad hoc comme celles qui verront le jour à Évry : le Génoscope-Centre national de séquençage (CNS) et le Centre national de génotypage ( $\mathrm{CNG}$ ). C'est à la suite de ces décisions que fut lancé le projet Genopole. Tout commence avec la découverte de l'ADN...

10 Conséquences du développement rapide de la recherche, ces deux grands instruments s'inscrivent dans les révolutions essentielles qui secoueront la biologie au cours de la seconde moitié du xxe siècle jusqu'au bouleversement très récent (1992) que constitue l'avènement de la génomique.

11 Tout commence en 1913 lorsque l'américain Thomas Morgan découvre que les chromosomes portent les gènes ${ }^{7}$. Trente ans plus tard, en 1944, le New-Yorkais Oswald Avery démontre que l'acide désoxyribonucléique ou $\mathrm{ADN}$, molécule quasi universelle dans le monde vivant porte l'information génétique de ces êtres vivants. Il fait la démonstration formelle de son rôle dans la transmission d'informations héréditaires. Tout s'enchaîne alors très vite. Le 25 avril 1953, un article des Britanniques James Watson et Francis Crick, paru dans la revue Nature, décrit pour la première fois la structure tridimensionnelle en double hélice de l'ADN. Celle-ci, véritable "mémoire " $\mathrm{du}$ vivant, renferme sous une forme chimique, au demeurant assez simple, l'ensemble des instructions élémentaires - ou gènes - nécessaires aux activités et au maintien de l'intégrité d'une cellule ou d'un organisme. La connaissance de ces instructions et surtout de leur combinatoire d'usage est indispensable à la compréhension des phénomènes biologiques au niveau cellulaire et moléculaire.

12 Ce n'est pourtant qu'en 1964 que des recherches montrent comment l'enchaînement linéaire des quatre constituants chimiques qui forment l'ADN peut déterminer la synthèse et surtout la structure d'une protéine ${ }^{8}$. Découverte fondamentale puisqu'au sein d'un organisme vivant, certaines protéines forment la charpente physique des cellules, d'autres leur permettent de fonctionner. Elles servent de matériau de construction, de récepteurs pour les signaux dans et entre les cellules et de catalyseurs pour les réactions biochimiques. Ainsi, dans bon nombre de maladies génétiques, une altération dans la structure d'un gène (mutation) se traduit par la synthèse d'une protéine défectueuse ou par son absence.

13 L'ADN apparaît alors comme « la molécule essentielle du vivant ». La fascination qu'elle exerce sur les chercheurs génère de nombreux travaux sur ses propriétés : comment se réplique-t-elle? Comment évolue-t-elle? De quelle manière diffère-t-elle d'une espèce 
à l'autre ? À l'occasion de ces recherches très fondamentales, les biologistes découvrent

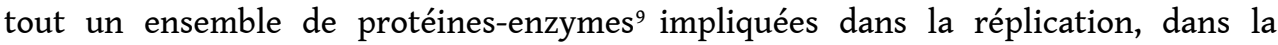
recombinaison ${ }^{10}$ et dans toutes les opérations moléculaires qui gravitent autour de l'ADN. Ces protéines deviennent alors autant d'outils potentiels pour faire in vitro ce que la cellule fait in vivo. Les premiers outils d'un véritable génie génétique ${ }^{11}$ sont ainsi découverts à la fin des années 1960. Ils vont permettre de comprendre l'intimité du fonctionnement du vivant, en particulier des gènes. Conséquence pratique, dès 1978, les biologistes utilisent des bactéries pour "fabriquer" des hormones humaines: insuline ou hormones de croissance, qu'aucun chimiste ne peut synthétiser. L'ère des biotechnologies nouvelles commence.

Les pionniers du séquençage

14 Dans les années 1970, ces recherches aboutissent à la constitution progressive de ce que j'appelle la "caisse à outils du vivant». Les outils moléculaires qui la composent sont souvent d'origine bactérienne comme les enzymes de restriction, sorte de «molécules ciseaux » capables de découper l'ADN au niveau de régions très spécifiques. Également très utilisés, les plasmides sont de petits fragments d'ADN circulaires qui se multiplient de façon autonome au sein d'une bactérie qui se divise ; dès les débuts de la génétique dite moléculaire, bactéries et plasmides servent de «tubes à essais vivants ». D'autres enzymes sont disponibles comme les ligases qui aident à recoller des fragments d'ADN double-brin ou les polymérases qui permettent de recopier une séquence d'ADN à l'identique. Les fragments d'ADN peuvent aussi être séparés par leur taille, grâce à un champ électrique généré dans un gel d'électrophorèse. Tout ceci permet, en fait, l'opération simplissime suivante: couper dans une molécule d'ADN d'une espèce donnée un petit morceau choisi à l'avance et situé à un endroit déterminé, pour le greffer dans une autre molécule d'ADN d'une espèce différente, à n'importe quel endroit. Tour de passe-passe rendu possible en raison de la nature même de l'ADN, molécule universelle du vivant.

15 Ces découvertes mènent naturellement à l'idée d'appréhender complètement toute l'information contenue dans l'ADN. Ceci passe par le déchiffrage chimique de l'enchaînement des quatre molécules de base (A, T, G, C), un peu ce que l'on fait lorsqu'on essaie de comprendre un livre, une phrase écrite dans un alphabet inconnu. L'opération s'appelle séquençage. Les premières méthodes permettant de "séquencer " datent de la fin des années 1970. La plus utilisée est celle du Britannique Frederick Sanger dite "séquençage enzymatique de Sanger " qui lui a valu son deuxième prix Nobel de chimie en 1980 avec P. Berg et W. Gilbert. Elle permet de déterminer l'arrangement linéaire des nucléotides $A, T, G, C$. Avec ces premières techniques, ont peut lire quelques centaines de ces molécules élémentaires. Au milieu des années 1980, ces méthodes utilisées de façon manuelle dans les laboratoires, commencent seulement à être automatisées...

En vérité, la plupart des chercheurs ne perçoivent pas les mutations profondes qui commencent à ébranler la recherche en biologie, véritable changement de paradigme dû, justement, au développement de ces technologies. À cette époque, étudier l'expression d'un gène unique occupe la majeure partie du travail d'un chercheur... et une partie de sa carrière! C'est le biologiste américain Ed Coe qui s'exclame : «Il y a tellement de gènes, et si peu de temps! ». Chaque gène à étudier demande, de surcroît, une approche adaptée: le scientifique est occupé à développer et optimiser les techniques; en réalité, il crée des instruments révolutionnaires. 
17 Un autre événement décourage la recherche à la fin des années 1980 : Japonais et Américains échouent dans leur tentative de séquençage $\mathrm{d}^{\prime} \mathrm{E}$. coli ${ }^{12}$. Les techniques utilisées ne sont pas encore assez mûres et les chercheurs n'ont pas intégré la nécessité d'organiser le travail de séquençage de manière industrielle. Les laboratoires travaillent encore de façon artisanale. Inutile, dans ce contexte, d'évoquer le séquençage du génome humain qui passe pour une entreprise réservée aux fous ou aux mégalomanes. Deux chercheurs vont, pourtant, imaginer un appareil qui rend possible un tel projet : en 1986, Leroy Hood et Lloyd Smith présentent la première machine de séquençage automatisée. Ce début de changement de paradigme, né de l'ambition de connaître l'ensemble de l'information génétique d'un individu, provoque une révolution dans la biologie qui va lancer les recherches du laboratoire Généthon, créé par l'AFM.

Le pas de géant du laboratoire Généthon

Trouver des solutions thérapeutiques pour traiter les maladies génétiques rares est la vocation première de l'AFM. Or, la découverte de ces molécules suppose de mieux connaître la maladie et donc le gène à l'origine de cette pathologie pour espérer le corriger directement ou améliorer le fonctionnement de la protéine défectueuse. Rechercher un gène dans l'ensemble de l'ADN humain revient à rechercher une ou quelques pages dans un livre d'un million de pages dont on ne connaîtrait à peu près rien.

19 Pour rechercher un gène, il fallait donc mieux connaître le génome de l'Homme. Sur ce dernier point, l'AFM, via son président de l'époque Bernard Barataud, trouve que les recherches ne vont pas assez vite, constat qui emporte alors la décision d'investir dans des recherches axées principalement sur une connaissance plus fine du génome de l'Homme. Le laboratoire Généthon voit le jour en 1990 grâce aux dons du Téléthon et l'appui de l'un de ses principaux inspirateurs, le prix Nobel Jean Dausset ainsi que de son élève Daniel Cohen. Ce dernier est convaincu que la seule manière de s'attaquer sérieusement au génome est de changer d'échelle dans la manière d'aborder ces questions. Il propose l'installation d'un laboratoire révolutionnaire, où plusieurs dizaines de machines fonctionnent en parallèle, où des automates remplacent des techniciens, le tout géré par une informatique de pointe, fonctionnant jour et nuit, 7 jours sur 7. Il va à contre courant de ce qui est usuel en matière de biologie à l'époque, même au États-Unis, en appréhendant le génome globalement, grâce à ces méthodes à grande échelle. Pour cela, il faut beaucoup d'argent. Le Téléthon et son succès arrivent fort à propos. Dans la foulée, Bernard Barataud attire un autre scientifique de renom, Jean Weissenbach, actuel directeur général du Génoscope. Leurs travaux permettent de produire en 1992 la première carte physique du génome humain ${ }^{13}$ et l'année d'après une carte génétique ${ }^{14}$ de seconde génération, toujours utilisée dans le monde entier pour accélérer la découverte des gènes impliqués dans les maladies génétiques. Le laboratoire du Généthon, né des efforts de l'AFM pour faire aboutir ses propres recherches, positionne la France au premier rang mondial dans la recherche en génomique. Surtout, il fonde une approche nouvelle qui aboutira à la création des deux grands instruments de la biologie en France.

Au milieu des années 1990, de grands équipements similaires apparaissent simultanément dans les grands pays de la recherche en biologie. Le premier d'entre eux est le Sanger Institute, centre de séquençage britannique créé en 1994 par une fondation privée, le Wellcome Trust. Aujourd'hui encore, le Sanger Institute reste le 
plus grand centre du monde réalisé dans l'esprit d'un TGE de la physique, dans la mesure où il prend complètement en charge des projets que des laboratoires seuls ne peuvent pas accomplir. Dans cette logique, le Wellcome Trust supporte l'essentiel des coûts du Centre et, en échange, le laboratoire travaille gratuitement pour les équipes de recherches dont les programmes ont été acceptés par le comité scientifique.

21 En France, ceci ne se faisait pas en biologie et ce n'est que lorsque l'AFM demanda à l'État, en 1994, de prendre le relais de son action, que le ministère de la Recherche décida, en 1996, de créer le Centre national de séquençage et, en 1998, le Centre national de génotypage. Ce sont les deux premiers grands instruments de la recherche en biologie, premiers " édifices " proches de ce qui se faisait en physique. à ceci près que, lors de leur création, leurs budgets respectifs ont été pris en charge, non par le CNRS mais directement par le ministère de la Recherche, c'est-à-dire l'État. Ces TGE de la biologie à grande échelle ${ }^{15}$ ont conservé leur lien direct avec le ministère, c'est-à-dire qu'ils reçoivent des moyens leur permettant à la fois de se gérer et d'acquérir les outils dont ils ont besoin. Ils disposent donc d'une certaine indépendance financière... qui échappe dans ce cas au CNRS et à l'Inserm. Notons, sans le commenter, que pendant cette même période, le CNRS acceptera de participer activement à deux autres grands projets de TGE pour la physique : Virgo et Soleil.

La révolution conceptuelle de la biologie à grande échelle

Ces grands instruments s'inscrivent pourtant dans un processus qui n'est pas simplement de doter la recherche de moyens financiers protégés. C'est aussi une manière de participer à une évolution qualitative très forte qui peut aboutir à des révolutions conceptuelles et médicales dont on imagine encore difficilement aujourd'hui les conséquences pour demain. Ces technologies nouvelles vont sortir la biologie moléculaire de l'une des critiques essentielles et légitimes qui lui était faite: son réductionnisme. L'une des principales attaques reposait sur la manière de généraliser les résultats : comment peut-on comprendre le vivant et sa complexité en observant le fonctionnement d'un seul gène? Cela revient à vouloir comprendre la complexité de la sociologie humaine en étudiant un individu particulier, ce qui n'a guère de sens. Toutes ces critiques étaient fondées mais la biologie moléculaire était très réductionniste car... elle ne savait pas faire autrement! Ainsi, la mission du Centre national de séquençage - Génoscope - qui est de produire et d'interpréter de grands volumes de données de séquences de haute qualité, a fait basculer le travail dans une dimension supérieure.

Des centres de séquençage de l'ADN comme le Génoscope, véritable usine à produire des séquences, sont nécessaires car il est impossible de lire d'une traite la séquence des immenses molécules d'ADN - les chromosomes ${ }^{16}$ - qui renferment l'information héréditaire d'un organisme. Les chromosomes humains contiennent des molécules d'ADN longues de plusieurs dizaines à plusieurs centaines de millions d'éléments. Or, chaque opération élémentaire de séquençage ne livre qu'un fragment de 500 à 1000 éléments. Il faut donc en réaliser un très grand nombre et même produire un volume de séquences plusieurs fois supérieur à la taille du chromosome, ces lectures répétées permettant de raccorder les séquences les unes aux autres et de s'assurer de la qualité $\mathrm{du}$ résultat de chaque lecture individuelle. Déterminer la séquence complète d'un chromosome humain nécessite ainsi des dizaines de millions de lectures. L'opération s'apparente à la reconstitution d'un livre dont on aurait découpé les milliers de pages 
en petits morceaux de 500 lettres, à la différence près que, dans l'ADN, il n'y a ni virgule, ni point, ni renvoi à la ligne, ni paragraphe visible ou aisément décelable.

Avant le séquençage total du génome humain, quelques gènes avaient été identifiés et concernaient, pour la plupart, les maladies génétiques rares dites monogéniques car conséquences de la mutation ou de la modification intervenant sur un seul gène, comme la myopathie, la mucoviscidose ou l'hémophilie. Or, les technologies dites de la biologie à grande échelle permettent de connaître la structure de milliers de gènes de diverses espèces, de suivre le fonctionnement de 20000 gènes en même temps et d'inventorier un très grand nombre de protéines présentes dans une cellule, c'est-àdire d'acquérir un jour la connaissance quasi exhaustive du génome et des molécules du vivant. L'idée n'est pas seulement de faire beaucoup plus et d'aller plus vite, mais d'avoir un regard sur le vivant plus proche de la réalité. Et cela est capital. Si au lieu d'observer l'expression d'un seul gène dans une cellule, nous sommes capables d'étudier la manière dont tous ces gènes interagissent dans le temps, nous aurons une représentation plus fidèle de la réalité et du fonctionnement du vivant. De la même façon, si nous pouvons répertorier à un moment donné toutes les protéines (ou presque) présentes dans une cellule, nous aurons une idée beaucoup plus précise de leur fonctionnement.

Le séquençage : artisan de nouvelles techniques moléculaires

Ces efforts de séquençages systématiques des génomes de virus, de bactéries et d'organismes dits supérieurs, dont l'Homme, ont conduit à développer les techniques d'acquisition de données moléculaires. Autrement dit, les grands projets de séquençage du génome ont permis de découvrir de nouvelles technologies moléculaires, sorte d'iceberg dont la partie visible est aujourd'hui la génomique structurelle et fonctionnelle ${ }^{17}$. Demain, la connaissance de la partie immergée mettra à jour un changement de paradigme sur les aspects fondamentaux et appliqués de la biologie. Il s'agit, en fait, d'une nouvelle vision du vivant dans ses aspects les plus intimes, dont les techniques moléculaires et informatiques à grand débit sont les instruments.

Au niveau du génome, les puces à $\mathrm{ADN}^{18}$ permettent déjà d'appréhender une dimension nouvelle. Si la génétique permet d'isoler un gène à fonction connue et de l'étudier au niveau moléculaire, la génomique livre une pléthore de gènes dont il faut déterminer les fonctions et les interactions. Ces deux approches ne sont pas antinomiques mais complémentaires.

L'étude du transcriptome ${ }^{19}$ est réalisée via des " puces ", ou d'autres méthodes à grand débit comme le $\mathrm{Sage}^{20}$, capables de détecter l'expression de la quasi totalité des gènes d'un organisme à analyser. La génomique permet même de suivre l'expression de tous ces gènes dans le temps, avec de surcroît, les niveaux d'expression de chaque gène interdépendant. Ainsi, toutes ces techniques moléculaires à grand débit comme les puces à $\mathrm{ADN}$ ou le procédé de réaction de polymérisation en chaîne (PCR) qui permet de reproduire à volonté et en très grand nombre des fragments d'ADN à partir d'une quantité infime de matériel, modifient le contexte actuel des projets de recherche dans les sciences médicales et la biologie moléculaire humaine.

Les fondations d'une nouvelle médecine

Ces recherches prendront toute leur ampleur en médecine et, par exemple, en cancérologie, maladie qui affecte la croissance et les propriétés « sociales » des cellules normales. L'organisme assure un contrôle strict des multiplications cellulaires. Pour diverses raisons complexes, il peut arriver qu'une cellule échappe à la vigilance de 
notre organisme. Ce phénomène, rare, va entraîner une multiplication incontrôlée et anarchique des cellules, aboutissant à ce que l'on appelle une tumeur locale puis à un cancer généralisé lorsque cette tumeur essaime dans l'organisme.

Si nous prenons pour exemple la différence entre les recherches que nous développions en cancérologie il y a vingt ans et ce que l'on fait aujourd'hui grâce à ces nouvelles technologies, le fossé est immense. À l'époque, notre travail visait à tenter d'identifier à l'intérieur d'une cellule cancéreuse, humaine ou animale, les gènes altérés. Quand on avait caractérisé l'un de ces gènes, nous étions très satisfaits, deux gènes très, très heureux, et au troisième gène isolé, nous étions tellement fatigués que l'on passait à autre chose... Le travail n'était pourtant pas fini... Lorsqu'on avait isolé un gène dont on savait pertinemment qu'il était modifié dans une cellule cancéreuse, il fallait essayer de comprendre sa fonction, son mécanisme d'action, comment son altération pouvait aboutir à une croissance anormale et en quoi il pouvait être impliqué dans d'autres types de cancers. Les quinze ans qui ont suivi la découverte des premiers "gènes de cancers » ont été consacrés à cette analyse méthodique et systématique très longue. Le but de cet archivage laborieux était de déterminer les gènes les plus importants. Venaient ensuite d'autres études: si ce gène est altéré dans cette tumeur précise, le pronostic vital est-il plus grave ou moins grave? Quels traitements peut-on imaginer contre ce gène, son produit, son action? Tout un ensemble de recherches se concentraient donc autour d'un gène, d'une mutation dont on essayait, bien entendu, de déduire des connaissances générales. Tâche très difficile car sur 100 tumeurs du sein d'apparence semblable, 20 possédaient un gène muté alors que dans 20 autres, le gène n'était pas muté mais seulement fortement exprimé. Tout ceci nous amenait à une situation extrêmement complexe et paradoxale avec d'un côté, des recherches fondamentales très nombreuses, de qualité exceptionnelle et très pointues en cancérologie moléculaire fondamentale touchant à la biologie générale du cancer, et de l'autre, des médecins qui nous reprochaient le peu de progrès des traitements. On en était là jusqu'en 1995.

Or, à partir de ces nouvelles technologies, l'identification possible dans la tumeur d'un nombre important d'altérations génétiques devient possible. Ce n'est pas encore simple à faire mais le processus est en cours. De toute évidence, si cet inventaire devient réalisable, la recherche disposera d'un moyen d'établir un portrait moléculaire de la tumeur beaucoup plus complet qu'en observant un seul gène.

31 Cette vision globale autorise une question fondamentale à laquelle les chercheurs étaient incapables de répondre auparavant : les lésions situées dans la tumeur primaire, tumeur du sein par exemple, sont-elles maintenues dans les tumeurs secondaires, les métastases, ou ont-elles déjà évolué ? Dans la foulée, une autre question essentielle devient possible : quels gènes sont exprimés dans cette tumeur à un moment donné ? Si l'on reprend l'exemple des 100 tumeurs du sein très semblables, on trouve que le profil d'expression des gènes n'est pas le même dans toutes les tumeurs, bien que les cellules concernées paraissent morphologiquement identiques. Ceci permet d'établir une forme de classification nouvelle et surtout de rechercher une corrélation entre l'expression des gènes dans une tumeur et la réponse au traitement.

Ceci aboutit à un regroupement de tumeurs apparemment de même type en sousfamilles et montre que l'on obtient $90 \%$ de bonnes réponses au traitement pour certaines de ces sous-familles alors que pour d'autres, le résultat est quasi nul. Cette observation permet de préciser les traitements adaptés à certains types de tumeurs et 
d'exclure celles qui ne s'y prêtent pas. L'un des objectifs est d'épargner à des patients atteints de ces lésions un traitement agressif inutile; un autre est de définir une stratégie visant à trouver d'autres thérapeutiques pour traiter ce type de cancer. À cette observation globale touchant les cellules malades, on peut adjoindre une recherche des caractéristiques génétiques de chaque malade. On sait d'ores et déjà que les traitements seront dans l'avenir optimisés par cette double approche : connaissance des caractéristiques génétiques de la tumeur, connaissance des caractéristiques génétiques du malade. Ces technologies permettent donc de définir de nouvelles voies thérapeutiques qui vont accroître l'efficacité du traitement chez des patients atteints du même type de lésions. Or, cette façon d'aborder la médecine est directement liée à la biologie à grande échelle et sans les grands instruments, comme le CNS et le CNG, on ne serait jamais parvenu à ces résultats.

La nouvelle dimension génétique de l'Homme et de ses pathologies : les besoins en génotypage

Cette nouvelle dimension de l'Homme démontre ce que nous savons tous intuitivement: nous sommes tous inégaux face aux maladies. Dans un environnement déterminé, chaque individu aura une réponse différente. Certains, par exemple, fument un paquet de cigarettes par jour et développeront des pathologies rapides, bronchite chronique, allergies ou cancer alors que d'autres gros fumeurs vivront en apparente bonne santé pendant très longtemps.

En octobre 1998, le CNG, actuellement dirigé par Mark Lathrop, a repris les activités génomiques de génotypage de Généthon et son objectif est, notamment, l'identification de gènes morbides impliqués dans les maladies fréquentes. Le CNG participe, par exemple, à la réalisation de projets européens de grande envergure tels ceux visant à étudier l'hypertension, le diabète et la compatibilité HLA ${ }^{21}$ dans les greffes de moelle. Aujourd'hui, nous avons, en effet, une certitude : dans toutes les pathologies humaines, une composante génétique explique, en partie, notre inégalité face à ces pathologies. C'est ce que l'on appelle les gènes de prédisposition qu'il ne faut surtout pas réduire à une vision simpliste car dans ce domaine, il faut appréhender les comportements, les environnements mais également la complexité de l'ensemble du génome. Avoir un gène de prédisposition ne signifie pas que l'on sera malade. Cela indique seulement un risque plus ou moins élevé par rapport au risque moyen.

C'est à ce niveau que les recherches du Centre national de génotypage deviennent essentielles. Le génotypage étant la caractérisation de l'ensemble des différences existant entre les génomes, d'un individu à l'autre, ces recherches visent à établir des corrélations entre la présence dans le génome de différents individus de certains allotypes, c'est-à-dire de certaines allèles ${ }^{22}$ de gènes à des endroits déterminés par rapport à la fréquence d'une pathologie donnée. Un allèle est une version possible naturelle d'un gène. Or, n'oublions pas, à ce stade, que tout individu résulte d'une construction faite par les réunions d'une moitié de patrimoine génétique issue de sa mère et d'une autre moitié de son père. Nous résumons cela en disant que, chez un individu, chaque gène est présent en deux exemplaires, l'un sur le chromosome d'origine paternelle, l'autre sur le chromosome d'origine maternelle, situés au même endroit (dans chaque chromosome). Ces deux allèles sont soit identiques à un endroit donné, l'individu est alors homozygote à cet endroit pour ce gène, soit différents dans leur composition, l'individu est alors hétérozygote pour ce gène. L'objectif du CNG est de fournir une assise scientifique solide à des affirmations de ce type: risques de 
maladies cardiovasculaires accrues, par exemple, en raison de la présence de deux allèles particuliers. Le CNG est alors capable de comparer massivement et de manière parallèle, le génome de milliers d'individus et d'essayer de discerner, parmi ces différences, celles qui sont impliquées dans des pathologies comme l'obésité, le diabète, les maladies cardiovasculaires, les allergies ou le cancer.

Ces recherches ont ainsi permis d'élaborer une nouvelle méthodologie, la génomique structurelle et fonctionnelle, née de la génétique moléculaire et de l'informatique. C'est, en effet, la robotisation et la taylorisation des différentes procédures utilisées couramment dans les laboratoires de biologie moléculaire qui ont facilité sa conception, ainsi que la miniaturisation des supports due au développement des microtechnologies, demain les nanotechnologies. Miniaturisation qui permettra sans doute de concevoir des laboratoires d'analyses moléculaires portables... Alors que les équipements de la recherche en physique sont condamnés à toujours plus de gigantisme, la biologie entrevoit, elle, son avenir à l'échelle de la cellule, par le biais des nanotechnologies...

\section{NOTES}

1.Le grand accélérateur européen de particules a été construit et est exploité par une organisation connue sous le nom de Cern, le Centre européen de la recherche nucléaire, qui vient de fêter ses 50 ans. Si son siège se trouve à Genève, ses installations se répartissent de part et d'autre de la frontière franco-suisse, sur le territoire genevois et dans le Pays de Gex. Le Cern a le statut d'organisation internationale, ce qui signifie qu'il est financé par les contributions des États membres de la Communauté européenne qui fournissent également les quelque 3500 personnes qui y travaillent. Actuellement, le synchrotron à protons du Cern $(400 \mathrm{GeV})$ est l'un des plus puissants du monde, juste derrière celui de Fermilab aux États-Unis (500 GeV).

2.Soleil est un accélérateur de particules qui produit un rayonnement synchrotron permettant d'explorer la matière, inerte ou vivante. Ce rayonnement est fourni par un anneau de stockage de $354 \mathrm{~m}$ de circonférence dans lequel des électrons de très haute énergie circulent quasiment à la vitesse de la lumière. Situé sur le Plateau de Saclay en Essonne et opérationnel à partir de 2006, Soleil ouvre de nouvelles perspectives pour sonder la matière avec une résolution de l'ordre du millionième de mètres et une sensibilité à tous les types de matériaux. Ses applications seront multiples en physique, en chimie, en sciences de l'environnement, en médecine et en biologie.

3.L'expérience franco-italienne Virgo repose sur un interféromètre géant de $3 \mathrm{~km}$ de long et installé à Cascina près de Pise en Italie. Son ambition est de détecter les ondes gravitationnelles produites par de violents phénomènes cosmiques. La construction de l'ensemble de l'interféromètre vient de s'achever. Les premiers tests de Virgo sont en cours et la prise de données scientifiques devrait démarrer en 2005.

4.Rapport de janvier 2001 sur « Le rôle des très grands équipements dans la recherche publique ou privée, en France et en Europe ». L'Office parlementaire d'évaluation des choix scientifiques et technologiques a été créé par la loi nº 83-609 du 8 juillet 1983, à la 
suite d'un vote unanime du Parlement. Aux termes de la loi, sa mission est « d'informer le Parlement des conséquences des choix de caractère scientifique et technologique afin, notamment, d'éclairer ses décisions ». à cet effet, l'office « recueille des informations, met en œuvre des programmes d'études et procède à des évaluations ». 5.Ibid.

6.La génomique est la science qui se propose d'étudier la structure, le fonctionnement et l'évolution des génomes.

7.Le gène est un fragment d'ADN qui code pour une protéine. Il peut comporter de quelques centaines à plusieurs centaines de milliers de nucléotides. Chaque gène comprend fondamentalement quatre régions : un promoteur, une séquence codante, un terminateur, des séquences de régulation.

8.L'ADN est un polymère formé de deux chaînes formant une double hélice. Quatre molécules élémentaires nommées nucléotides se succèdent linéairement sur chacune de ces chaînes, qui prennent ainsi la forme d'un très long collier composé de quatre perles différentes, symbolisées par les lettres A, T, G et C. L'ordre de ces nucléotides détermine la signification du message codé par la molécule (gène). En effet, cette molécule est transcrite point par point sous forme d'acide ribonucléique (ARN). Certaines de ces molécules d'ARN servent de messager (ARN messager) pour être traduites en protéines.

9.Les enzymes sont des protéines spécifiques qui catalysent les réactions biochimiques de l'organisme.

10.La recombinaison génétique concerne tout processus permettant d'obtenir un assemblage nouveau d'informations génétiques. Ainsi, la recombinaison méiotique qui a lieu lors de la méiose-division cellulaire qui aboutit à la formation des gamètes est produite par un mécanisme dans lequel les chromosomes issus du père et de la mère échangent réciproquement certains segments de leur matériel génétique.

11.Le génie génétique est la partie de la biologie qui utilise les outils de la biologie moléculaire pour modifier la structure ou le fonctionnement d'un ou de plusieurs gènes. Par exemple, certains segments de chromosomes contenant un gène d'intérêt peuvent être isolés et intégrés au génome d'autres organismes : bactéries, levures, plantes... Le génie génétique permet ainsi d'obtenir, à partir de bactéries, la synthèse et la fabrication industrielle de substances comme les hormones ou les vaccins.

12.Escherichia coli est un genre bactérien dans lequel on ne retrouve qu'une seule espèce. Sa culture très facile et sa grande tolérance de variation de $\mathrm{pH}$ expliquent son utilisation récurrente dans les manipulations en biologie.

13.La carte physique du génome de l'Homme est la représentation de la disposition des gènes sur les chromosomes, obtenue en utilisant directement le fragment d'ADN qui le contient.

14.La carte génétique du génome de l'Homme est la représentation de la disposition des gènes le long de la molécule d'ADN, utilisant un ensemble de repères moléculaires identifiés et positionnés le long de chaque chromosome. Ces repères - marqueurs permettent de localiser plus facilement les gènes sur les différents chromosomes. 15.La biologie à grande échelle est un ensemble de techniques permettant d'accélérer l'acquisition et l'analyse des résultats. Elle implique une automatisation des procédures expérimentales en biologie, grâce à l'utilisation de robots, la mise en parallèle d'appareils d'analyses, le tout placé sous contrôle d'une importante informatique de gestion et d'exploitation et de procédures d'assurance qualité. 
16.Les chromosomes sont la forme visible au microscope optique que prend la molécule d'ADN condensée, associée à des protéines pendant la phase de division cellulaire. 17.La génomique fonctionnelle concerne la partie de la génomique qui étudie la fonction des protéines codées par un gène, leur régulation et leurs interactions. 18.Les puces à ADN sont des supports solides - petite lame de verre comme celles utilisées en microscopie traditionnelle ou membrane de nylon - sur lesquels des milliers de fragments d'ADN sont déposés de façon géométrique à l'aide d'une micropipette robotisée. Grâce à cette technique, chacun des fragments d'ADN est représenté par un point (ou puce) sur le support. Ils servent de sondes pour fixer de façon très spécifique les fragments de gènes complémentaires (cibles), présents dans les échantillons biologiques à tester.

19.La transcriptomique est l'étude de l'expression totale des transcrits (ARNmessagers) des gènes dans une cellule, un tissu ou un organisme donné.

20.La méthode à haut débit Sage (Serial Analysis of Gene Expression) permet une analyse de la fréquence d'un ARN messager (l'ARNm étant le produit de la transcription de l'ADN, en clair lorsque l'ADN est converti en ARN), quel que soit son niveau d'expression parmi les milliers produits dans une cellule à un moment donné.

21.Les molécules HLA sont des présentoirs pour les déterminants antigéniques. Les chaînes polymorphes qui composent les molécules HLA sont codées par un ensemble de gènes situés sur le chromosome 6 , ensemble de gènes très proches les uns des autres. De ce fait, les gènes HLA sont transmis en bloc (haplotype) des parents aux enfants. Chaque enfant hérite d'un haplotype paternel et d'un haplotype maternel. Chaque allèle de chacun des 2 haplotypes est exprimé. L'identification de ces allèles correspond au groupage HLA.

22.Allèle : contraction d'allélomorphe. Version particulière d'un gène porté sur l'un des deux chromosomes homologues d'une cellule, l'un venant du père, l'autre de la mère.

\section{RÉSUMÉS}

The Molecular Biology Large Equipments, Beginnings of the Tomorrow Medicine Essentially historical, the concept of very large scientific equipments finds its origin in the particle physics large apparatus. Since 1995, it concerns only physics and universe sciences. With the discovery of DNA in 1953, the emergence of genomics developed the necessity for large biology equipments leading to the creation of the national sequencing and genotyping centers. DNA is going to exert such a fascination on researchers worldwide that a lot of laboratories will concentrate their research on this molecule. At the end of the 70's, creation of genetic engineering tools will foster understanding of gene action. These discoveries will necessarily converge in the project of the sequencing of all information contained in DNA. The first efficient sequencing method is due to Frederick Sanger but this method will remain at a very small scale. But in 1986, Leroy Hood and Lloyd Smith, two brilliant researchers, imagine the first automatic sequencer. This tremendous evolution will induce a revolution in biology which will mark the research of Genethon, laboratory of the French Association of Muscular Dystrophy. Their research allowed the publication of the physical map of the human genome in 1992 and of the genetic map in 1993. As in the physics field where practical experiments are almost impossible to 
realise in laboratory, these new tools lead to the creation of two large facilites in biology: the national sequencing center and the national genotyping center.

Genomics also study the expression of all the genes. It concerns in fact, a new vision of most intimate aspects of the living being that offer conceptual and medical revolutions of which the instruments are high speed data processing and molecular techniques. This new dimension of man and his pathologies will lead to an individual medicine where the study of the human genome will help to deal more specifically with the patient and the increase of the efficiency of a treatment on patients affected with the same disease.

\section{INDEX}

Mots-clés : biologie moléculaire, Téléthon, AFM, Génopole, gène, bioparc, génome, biotechnologies, ADN, séquençage, pathologie, génotypage

\section{AUTEUR}

\section{PIERRE TAMBOURIN}

Pierre Tambourin, directeur général depuis son lancement en 1998 de Genopole à Évry est un ancien élève de l'École polytechnique dont la spécialité en génétique moléculaire l'a naturellement conduit à effectuer et diriger des recherches à l'Inserm pendant de nombreuses années. Essentiellement consacrés à la cancérologie expérimentale, ses travaux visaient à comprendre les mécanismes qui transforment une cellule normale en cellule cancéreuse, puis à identifier les gènes impliqués dans ces mécanismes. À partir de 1989, Pierre Tambourin troque la gestion de la cellule pour celle de l'humain et dirige jusqu'en 1992 la section de biologie de l'Institut Curie puis, de 1993 à 1997, le département des sciences de la vie du CNRS. 